\title{
Use of Natural Light vs. Cold LED Lighting in Installations for the Recovery of Victims of Gender Violence: Impact on Energy Consumption and Victims' Recovery
}

\author{
Raquel Amorim ${ }^{1}$, Juan Carlos López ${ }^{1,2}$, Valentín Molina-Moreno ${ }^{3}$ and \\ Antonio Peña-García ${ }^{1,2, *}$ \\ 1 Department of Civil Engineering, University of Granada, 18071 Granada, Spain; \\ raquelamorim@correo.ugr.es (R.A.); juancarloslopez@ugr.es (J.C.L.) \\ 2 Research Group "Lighting Technology for Safety and Sustainability”, University of Granada, \\ 18071 Granada, Spain \\ 3 Department of Management, University of Granada, 18071 Granada, Spain; vmolina2@ugr.es \\ * Correspondence: pgarcia@ugr.es; Tel.: +34-958-24-94-35
}

Academic Editor: Antonio Messineo

Received: 21 February 2017; Accepted: 2 April 2017; Published: 7 April 2017

\begin{abstract}
The efficiency of lighting installations is a major challenge concerning Governments, productive sectors and individuals. However, the importance of accurate lighting in some areas, especially those related to Health and Wellbeing is so critical that the constraints of energy efficiency and sustainability are not always a priority. This situation has become more critical with the current boom in the application of non-visual effects of light to these areas. In this study, the effects oftwo different kinds of lighting on femalevictims of gender violence are compared and analyzed in terms of positive results and impact on energy consumption and sustainability. The lighting technologies used are integrated in facilities where these women carry out different activities aimed at their integration into daily life after their traumatic experiences. The results are expected to become a tool for professionals working with these collectives and for installation designers. In spite of the well-known effects of cold light, especially for tasks involving arousal, sleepiness and other critical variables, it is demonstrated that daylight, which is obviously cheaper from productive and environmental perspectives, is better for this application.
\end{abstract}

Keywords: natural light; LED; energy savings; visual ergonomics; non-visual effects of light; victims' recovery

\section{Introduction}

How to improve the recovery of female victims of gender violence using the non-visual effects of light from a sustainable perspective will be analyzed. Although sustainability, non-visual paths and victims' recovery could seem independent topics, the impact of illumination on psychological and physiological parameters, via some non-visual paths such as melatonin or cortisol ones, can influence the way in which victims of gender violence get over traumatic situations. The remarkable impact of lighting in financial and environmental terms forms a complex polynomial, in which victims' recovery and wellbeing must come first and, once achieved, optimization must be sought.

\section{Non-Visual Effects of Illumination}

The use of the non-visual effects of light is a challenge to prevent and palliate several effects of violence [1] or feelings related with vulnerability [2]. Some of the effects of gender violence 
explained above (insomnia, depression, low self-esteem, isolation, etc.) are related with circadian rhythms, the non-visual paths of melatonin and, hence, the illumination conditions. Melatonin is a neurohormone secreted by the pineal gland which participates in processes such as sleep, relaxation and arousal [3]. The higher the level of melatonin, the greater the drowsiness. On the other hand, their inhibition leads to an alert being raised and insomnia. Cortisol [4], another hormone that also responds to light levels, has an antagonistic effect on melatonin: its release helps us to raise alertness levels. The inhibition of melatonin secretion and the release of cortisol are especially fostered when the levels of illumination are high and the color temperature of the light is cold.

Besides extreme or non-usual conditions of illumination, several works have demonstrated the influence of lighting in environmental or daily conditions on people's performance, behavior and well-being [5,6]. Thus, the study of Küller [7] about the impact of fluorescent light on endocrine, neurophysiological indexes on the level of well-being and stress, concluded that high illumination levels of fluorescent light can awaken the central nervous system and that this leads to stress. More recently, Hoffmann [8] concluded that workplace lighting and its variations are critical to determining the productivity of employees and their well-being. The results of Leichtfried [9] suggest that a brief exposure to bright light induced an increase in subjective mood and alertness, which dim light did not.

In this work, the impact of lighting on somnolence, mental effort, self-esteem and depression in women enrolled in programs to get over the consequences of gender violence will be studied. Departing from the results, some measures concerning the use of the light installations and power consumption will be proposed.

Due to the wide casuistry of traumas, previous experiences, age, social conditions and responses to therapy, it is necessary to remark that this research does not aim to find exact solutions to every problem, but to identify trends and potential lines of work that can contribute to the improvement of the quality of life of victims of gender violence, from a sustainable perspective if possible.

\section{Materials and Methods}

This study was conducted in the installations of the Andalusian Institute for Women (IAM) in Granada with women enrolled in workshops to get over traumatic experiences derived from gender violence. Questionnaires were conducted after ordinary workshop sessions. Their results were compared to quantitative measures of the lighting conditions in the rooms where the workshops took place.

\subsection{Participants and Sessions}

The participants of this study belonged to two groups: Group 1 and Group 2. Both groups were evaluated in two different sessions: S1, carried out under electrical lighting alone or complemented with natural light; and S2, exclusively under natural light. Both sessions were carried out at different times.

The participants were all victims of gender violence participating in group therapy. The average age was 41 according to the distributions by group and age ranges shown in Table 1 and Figure 1 . The women belonged to different social classes and were enrolled in the therapy to overcome the damage caused in their life.

Table 1. Age distribution in the different groups and sessions. Due to the variety of personal situations among the participants, not all of them assisted both sessions as seen in the differences in average age in each session within the same group.

\begin{tabular}{ccc}
\hline Group & Average & Standard Deviation (SD) \\
\hline 1-S1 & 41.64 & 9.54 \\
1-S2 & 39.50 & 9.62 \\
2-S1 & 41.90 & 10.13 \\
2-S2 & 41.00 & 9.01 \\
Total Sample & 41.08 & 9.3 \\
\hline
\end{tabular}




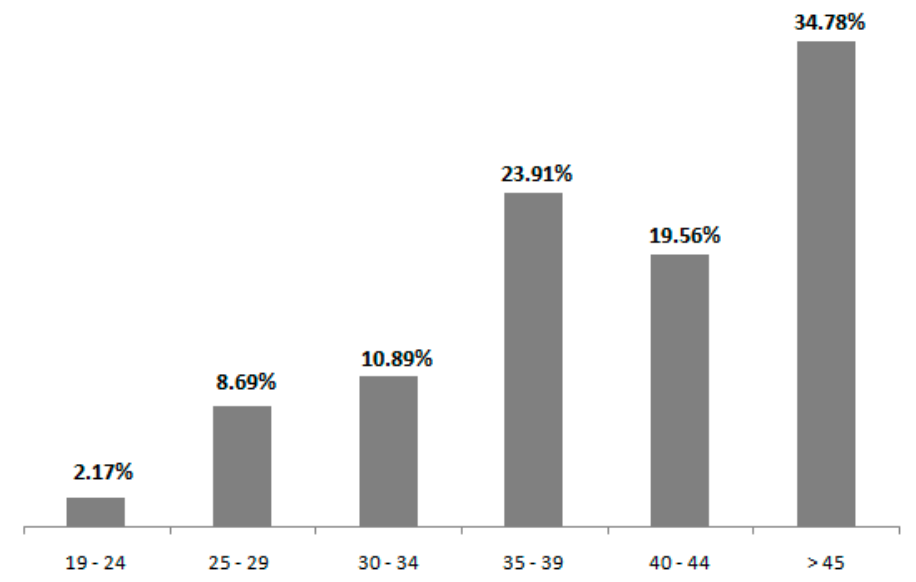

Figure 1. Age structure of Andalusian Institute for Women (IAM) users.

\subsection{Workshops and Workspace}

Therapy workshops were led by a psychologist of the IAM. They lasted two hours in the morning or afternoon schedule. Every user attendedthe workshops once a week, and groups were formed of 10 to 20 women.

Before beginning the questionnaires phase, the spaces and classrooms where the participants carry out their activities were evaluated. This evaluation included measurements of rooms dimensions, an inventory of luminaries (number, models' unitary power consumption), an inventory of windows allowing daylight entry and photometrical characterization. These spaces are the following:

\subsubsection{Auditorium}

It is a large room with the capacity to hold about 75 people, usually used for conferences and other events (Figure 2). Besides this use, some therapy sessions with the women participating in this study took place in this room. Although it has some windows, they look onto an extremely narrow street and they are always curtained. Hence, there is no natural light and any activity needs electrical lighting, which is provided by means of 15 luminaries, each with two 18W "LED tubes" having a correlated color temperature Tcc $=6000 \mathrm{~K}$. The installed power in the Auditorium is $540 \mathrm{~W}$.

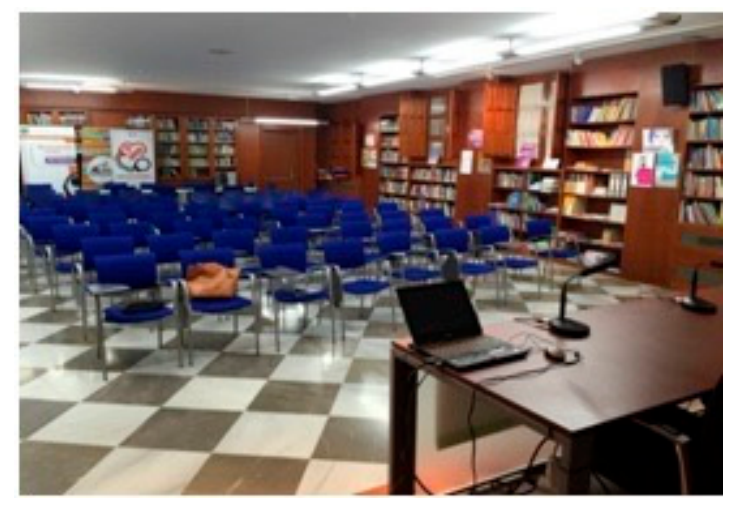

Figure 2. Auditorium in the Andalusian Institute for Women (IAM).

\subsubsection{Tower Room}

Other workshops and activities were carried out in one top-floor room in a tower (although the Institute has been completely redeveloped, the building is historical and some singular features such as the old tower are kept). It has the capacity to hold about 20 people. 
In this tower, there are wide windows allowing a high flux of daylight to enter (Figure 3). Depending on the time of the day, activities are carried out exclusively with natural light or with the combination of electrical and natural light. The lighting installation of this room consists of four luminaries, each with two 18W "LED tubes" having a correlated color temperature Tcc $=6000 \mathrm{~K}$. The installed power in the Tower room is $144 \mathrm{~W}$.

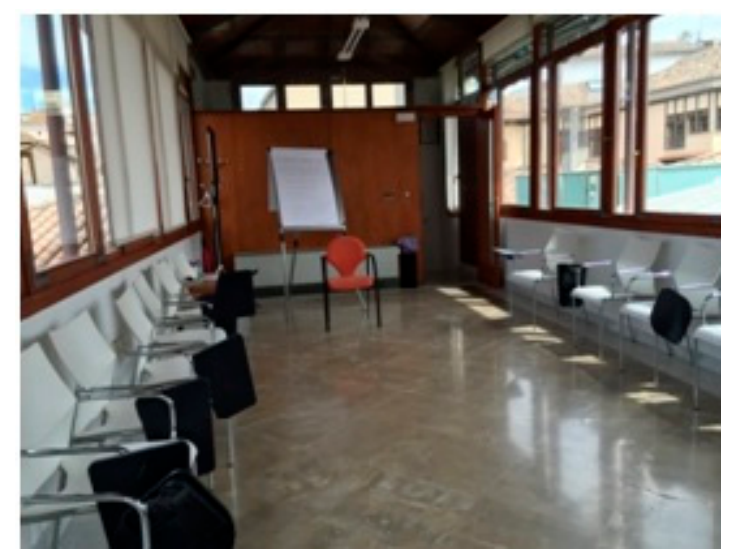

Figure 3. Tower room in the Andalusian Institute for Women (IAM).

\subsubsection{Questionnaire}

The questionnaire included seven parts: initial data (date, age, activity schedule); hours slept the night before; physical activity; drowsiness after activities are carried out (Karolinska Sleepiness Scale); effort needed to complete activities (Rating Scale for Mental Effort); self-esteem (Rosenberg scale) and Satisfaction With Life Scale (SWLS).

These scales, that are well-known and fully validated by the scientific community, have the following particularities:

Drowsiness: Karolinska Sleepiness Scale

The Karolinska Sleepiness Scale (KSS) is used to indicate the degree of drowsiness of the individual after completing one task [10]. It ranges from 1 to 9 between two extremes: Awake (1) and falling asleep (9).

\section{Mental Effort: Rating Scale for Mental Effort}

The Rating Scale for Mental effort (RSME) evaluates the effort needed to complete one given task [11]. It ranges from 0 to 150 including some labels indicating the degree of effort, from "absolutely no effort" to "extreme effort".

Self-Esteem: Rosenberg Self-Esteem Scale

The Rosenberg Self-Esteem Scale evaluates self-esteem, understood as feelings of self-worth and self-respect [12]. It consists of 10 questions, with five being set positively and five negatively so as to control the effect of self-administered acquiescence.

\section{Satisfaction with Life Scale (SWLS)}

The purpose of this scale is to assess the cognitive aspects of the welfare of a person [13]. It has five options which are assessed as agree or disagree statements within the Likert 7-point scale. 


\subsection{Measurements of Lighting Conditions}

The spaces under consideration were illuminated with natural light, LED luminaries or the combination of both. The light emitted by the LED luminaries was cold, with a correlated color temperature.

Average illuminance, and illuminance uniformity, were measured with a calibrated luxmeter-colormeter on the working planes of the rooms under consideration. The rooms had opaque curtains that can be open or closed, allowing daylight to enter or not. These condition are shown in Table 2.

Table 2. Data collection and space conditions.

\begin{tabular}{cccccc}
\hline Group & Session & Electrical & Illumination Conditions & Eav (Lux) & $\mathbf{U}_{\mathbf{0}}$ \\
\hline 1-S1 & Afternoon & on & LED + natural & 4029 & 0.529 \\
1-S2 & Afternoon & off & Natural & 3881 & 0.462 \\
2-S1 & Morning & on & LED & 506 & 0.623 \\
2-S2 & Morning & off & Natural & 3881 & 0.462 \\
\hline
\end{tabular}

\section{Results and Discussion}

Analysis of the data shows that the physical activity (Table 3) and hours slept (Figure 4) are quite similar between groups and sessions. This eliminates some potential biases due to lack of homogeneity among groups independent from the lighting conditions.

Table 3. Physical activity in general terms.

\begin{tabular}{ccccc}
\hline Physical Activity & 1-S1 & 1-S2 & 2-S1 & 2-S2 \\
\hline Regular practice & YES & YES & YES & YES \\
Type of activity & Walk & Walk & Walk & Walk \\
\hline
\end{tabular}

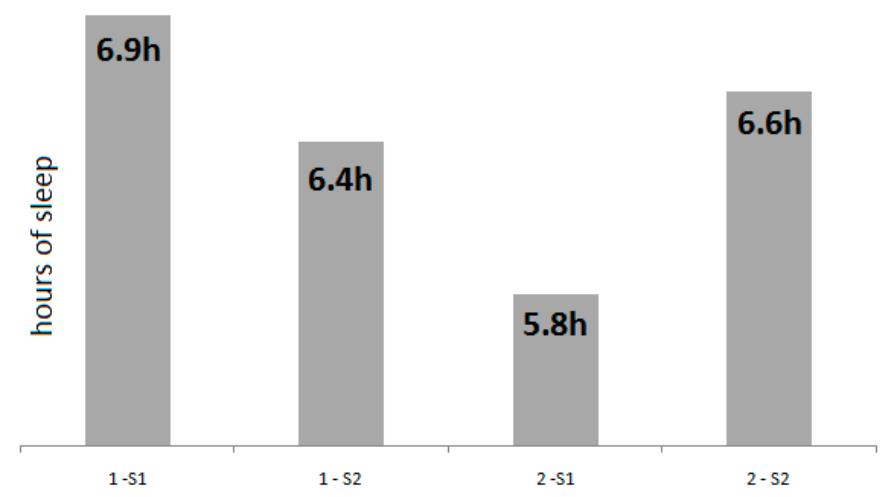

Figure 4. Slept hours.

In addition, the reported hours slept are accurate, which eliminates impaired performances due to conditions external to the activities in the classrooms such as psychophysiological insomnia. This kind of insomnia is a behavioral disorder concerning the inability to sleep and its consequences include trauma, worries, and sleepiness. This last consequence increases distraction during the execution of a monotonous task and impairs the performance of psychomotor tasks [14,15]. Table 4 presents a drowsiness estimation after the workshops measured with the Karolinska Sleepiness Scale (KSS). 
Table 4. Karolinska Sleepiness Scale (KSS) average scores and standard deviation in the different groups and all the participants together.

\begin{tabular}{ccc}
\hline Group & Av. Score & SD \\
\hline 1-S1 & 1.5 & 1.28 \\
1-S2 & 2.0 & 1.33 \\
2-S1 & 1.8 & 1.25 \\
2-S2 & 1.4 & 0.93 \\
Total Sample & 1.6 & 1.15 \\
\hline
\end{tabular}

Analysis of Table 4 shows that the scores in the KSS are quite similar in all the groups. This result is important because, in spite of the different lighting conditions, going from 500 to 4000 lux, there seems to be no difference in drowsiness as long as the standard recommendations are respected [16]. In fact, sessions in spaces with much lower average illuminance (2-S1) or any combination of light sources (LED+Sun in 1-S1, only sun in 1-S2 and 2-S2) show similar values in the KSS. This means that, in the absence of daylight, it is not necessary to consume high amounts of electrical power to keep the users awake when we are above the regulation requirements. In addition, LED sources have a similar impact on drowsiness to sun alone.

Concerning the mental effort, results are shown in Table 5:

Table 5. Rating Scale for Mental effort (RSME) average scores and standard deviation in the different groups and all the participants together.

\begin{tabular}{cccc}
\hline Group & Label & Av. Score & SD \\
\hline 1-S1 & almost no effort & 15.7 & 23.44 \\
1-S2 & a little effort & 19 & 29.98 \\
2-S1 & absolutely no effort & 6.3 & 15.01 \\
2-S2 & absolutely no effort & 3.6 & 9.24 \\
Total Sample & almost no effort & 11.3 & 21.14 \\
\hline
\end{tabular}

Analysis of Table 5 highlights differences among the scores depending on the group: although the mental effort is low in all the cases, the rates were remarkably higher in the first group. This result is noteworthy because the RSME average score in the first session of Group 2 (2-S1) is much lower than any of the average scores in Group 1, in spite of the remarkably lower illuminance (506 lux in Table 2). These results, combined with the values in Table 2, suggest that the higher effort could be due to two facts or the combination of both: (1) the sessions of Group 1 were carried out during the afternoon or (2) personal factors of the participants in Group 1. However, careful analysis of Table 1 and Figure 4 shows that eventual bias due to personal factors cannot come from the age of participants (similar in both groups) nor the habitual practice of exercise or hours slept (even higher in 1-S1, where the RSME score is high).

Furthermore, Table 1 shows that differences cannot come from the spectral distribution of the light used, because natural and LED illumination were present in both groups.

In summary, the levels of illumination do not seem to influence the mental effort of gender violence victims after their workshops, meaning that high consumptions of energy are not necessary for the facilities under consideration.

In addition, natural light seems to have similar effects to LED luminaries emitting cold light, which is deemed to enhance alertness and foster activity due to melatonin suppression. This last situation is similar to the one regarding somnolence. In addition, the most remarkable impact seems to come from the workshop schedule. These situations must be considered by designers and Public Administrations when planning these kind of installations. 
The third questionnaire concerned one extremely important parameter for female victims of gender violence: self-esteem. The results of participants' perception of this feeling after the workshops are presented in Table 6:

Table 6. Rosenberg scale of self-esteem average scores and standard deviation. Values for individual groups and all the participants together.

\begin{tabular}{cccc}
\hline Group & Label & Av. Score & SD \\
\hline 1-S1 & Average self-esteem & 26 & 7.08 \\
1-S2 & High self-esteem & 30 & 7.01 \\
2-S1 & Average self-esteem & 26 & 5.96 \\
2-S2 & Average self-esteem & 26 & 6.20 \\
Total Sample & Average self-esteem & 27 & 5.53 \\
\hline
\end{tabular}

According to the data above, only Group 1 experienced a real evolution in terms of self-esteem. This data is remarkable because this group achieved average somnolence and mental effort scores in the second session. Regarding the lighting conditions, the second session, in which self-esteem was improved, was carried out under natural light. This means that, besides the logical benefit of the workshop, the effect of daylight is at least similar to the effectof specific cold lighting, even if the levels of illuminance are lower (almost $4 \%$ less in S2). In Group 2, no improvement in self-esteem was observed from S1 to S2. This means that daylight is at least similar to cold LED light for the objectives of this research. In summary, besides the benefits in financial and environmental terms, the use of daylight in workshops with victims getting over the effects of gender violence seems to improve self-esteem or, at least, is no worse than cold LED light.

Regarding the Satisfaction with Life Scale (SWLS), the average scores and standard deviations are shown in Table 7:

Table 7. Satisfaction With Life Scale: average scores and standard deviation. Values for individual groups and all the participants together.

\begin{tabular}{cccc}
\hline Group & Label & Av. Score & SD \\
\hline 1-S1 & Below the average satisfaction & 17.8 & 6.7 \\
1-S2 & Below the average satisfaction & 16.9 & 7.65 \\
2-S1 & Dissatisfied & 14.5 & 7.45 \\
2-S2 & Below the average satisfaction & 16 & 5.94 \\
Total Sample & Below the average satisfaction & 16.2 & 6.91 \\
\hline
\end{tabular}

The values in Table 7 show low satisfaction with life, which might seem logical in victims of such traumatic situations. In addition, the evolution through the sessions is not significant. On one hand, this means that these workshops do not improve satisfaction with life and, on the other hand, the lighting conditions seem to be irrelevant in the improvement of this parameter. Although this last remark could lead one to understand that lighting is irrelevant, it has major importance because it means that, once more, natural light is at least as effective as specific white and cold LED lighting, with the subsequent impact on energy saving and the environment.

Once more, the efforts orientated towards fostering the use of natural light with light pipes, wide windows or other means seem to be profitable from all the perspectives.

In summary, concentrating all the workshops in the tower room at times when the users can profit from daylight could avoid the continuous use of one installation (the Auditorium) whose installed power is $540 \mathrm{~W}$. This means that $1.08 \mathrm{KWh}$ could be saved in each workshop. In a very conservative estimation, if only two sessions with victims of gender violence took place in the Auditorium every week for an estimated period of 11 months, a yearly saving of 207.36 KWh would be achieved with better results for the recovery of this extremely important collective. 


\section{Conclusions}

The results above show that the lighting installations in the facilities used for the recovery of female victims of gender violence do not need to consume high amounts of energy to achieve their objectives.

In spite of the heterogeneity of situations, the results obtained after coupling qualitative measures and quantitative answers to questionnaires suggest some important clues for the planning and management of lighting installations destined to activities of this important collective. The most remarkable are the following:

(1) Although the influence of cold light on arousal and concentration has been proven for years, the effect of natural light on sleepiness and mental effort is at least similar. This effect is independent of the levels of illuminance on the working plane, as long as the minimum values recommended by the international standards are fulfilled.

(2) Self-esteem is good on average. In addition, it was remarkably improved in one of the two groups when changing from electrical to natural lighting.

(3) The satisfaction with life of female victims of gender violence is, in general terms, low. It was not improved with the workshops; and the lighting conditions, according to the questionnaire about this topic, seem to have no influence.

(4) The most powerful effect on sleepiness and mental effort seems to come from the session (better in the morning), whereas the afternoon sessions seemed to improve self-esteem. Due to this encountered effect and the low influence of lighting, even when specific cold light luminaries were installed, it is recommended that the illumination is adapted to the most sustainable option in financial and environmental terms: the use of daylight is better than the use of electrical luminaries. When the absence of windows makes it difficult, the introduction of daylight with light-pipes or other reflecting and/or refracting systems should be considered.

(5) These considerations show that in some critical activities, such as the recovery of victims of gender violence, remarkable energy savings can be achieved even when the electrical lighting installation is sustainable as in the case presented in this research (100\% LED). In this case, the concentration of the workshops in areas and at times when daylight use is possible, allows important energy savings with better results for the planned task.

(6) The wide variety of variables that may influence any study with female victims of gender violence is a limitation. Hence, the search for optimal illumination that might improve some critical parameters related with better attention during the therapy workshops, better self-esteem, higher satisfaction with life and, in summary, a better quality of life, is a really complex problem. However, this complexity should not be an obstacle, but encourage multidisciplinary researchers to investigate the use of light to enhance wellbeing in this critical collective.

Acknowledgments: This work was conducted under funding from CAPES (Coordination of Improvement of Higher Education Personnel-Brazil) which has granted scholarships Doctoral number BEX 0947/14-8. The Andalusian Women's Institute (IAM) allowed the inventory and measurements on its installations, as well as the distribution of the questionnaires among the users that freely accepted to participate in this anonymous study. We especially thank the staff of the IAM (directives, psychologists, custodians and many other). Without their enthusiastic participation and help, this study would have never been possible.

Author Contributions: All the authors contributed equally to the research and redaction of the manuscript.

Conflicts of Interest: The authors declare no conflict of interest.

\section{References}

1. Amorim, R.; Molina-Moreno, V.; Peña-García, A. Proposal for Sustainable Dynamic Lighting in Sport Facilities to Decrease Violence among Spectators. Sustainability 2016, 8, 1298. [CrossRef]

2. Peña-García, A.; Hurtado, A.; Aguilar-Luzón, M.C. Impact of public lighting on pedestrians' perception of safety and well-being. Saf. Sci. 2015, 78, 142-148. [CrossRef] 
3. Cajochen, C. Alerting effects of light. Sleep Med.Rev. 2007, 11, 453-464. [CrossRef] [PubMed]

4. Figueiro, M.G.; Rea, M.S. Short-Wavelength Light Enhances Cortisol Awakening Response in Sleep-Restricted Adolescents. Int. J. Endocrinol. 2012. [CrossRef] [PubMed]

5. Suvanto, S.; Härma, M.; Lajtinen, J. The prediction of the adaptation of circadian rhythms to rapid time zone changes. Ergonomics 1993, 36, 111-116. [CrossRef] [PubMed]

6. Motohashi, Y. Alteration of circadian rhythm in shift-working ambulance personnel. Monitoring of salivary cortisol rhythm. Ergonomics 1992, 35, 1331-1340. [CrossRef] [PubMed]

7. Küller, R.; Wetterberg, L. Melatonin, cortisol, EEG, ECG and subjective comfort in healthy humans: Impact of two fluorescent lamp types at two light intensities. Lighting Res. Technol. 1993, 25, 71-80. [CrossRef]

8. Hoffmann, G.; Gufler, V.; Griesmacher, A.; Bartenbach, C.; Canazei, M.; Staggl, S.; Schobersberger, W. Effects of variable lighting intensities and colour temperatures on sulphatoxymelatonin and subjective mood in an experimental office workplace. Appl. Ergon. 2008, 39, 719-728. [CrossRef] [PubMed]

9. Leichtfried, V.; Mair-Raggautz, M.; Schaeffer, V.; Hammerer-Lercher, A.; Mair, G.; Bartenbach, C.; Canazei, M.; Schobersberger, W. Intense illumination in the morning hours improved mood and alertness but not mental performance. Appl.Ergon. 2015, 46, 54-59. [CrossRef] [PubMed]

10. Akerstedt, T.; Gillberg, M. Subjective and objective sleepiness in the active individual. Int. J. Neurosci. 1990, 52, 29-37. [CrossRef] [PubMed]

11. Zijlstra, F.R.H. Efficiency in Work Behavior: A Design Approach for Modern Tools. Ph.D. Thesis, Delft University of Technology, Delft, The Netherlands, 1993.

12. Schmitt, D.P.; Allik, J. Simultaneous administration of the Rosenberg Self-Esteem Scale in 53 nations: Exploring the universal and culture-specific features of global self-esteem. J. Personal. Soc. Psychol. 2005, 89, 623. [CrossRef] [PubMed]

13. Diener, E.D.; Emmons, R.A.; Larsen, R.J.; Griffin, S. The satisfaction with life scale. J. Personal. Assess. 1985, 49, 71-75. [CrossRef] [PubMed]

14. Porcu, S.; Bellatreccia, A.; Ferrar, M.; Casagrande, M. Sleepiness, alertness and perfomance during a laboratory simulation of an acute shift of the wake-sleep cycle. Ergonomics 1998, 41, 1192-1202. [CrossRef] [PubMed]

15. Anderson, C.; Horne, J.A. Sleepiness enhances distraction during a monotonous task. Sleep N. Y. Westchest. 2006, 29, 573.

16. European Committee for Standardization. European Standard EN 12464-1:2011 (Lighting of Work Places. Part 1: Indoor Work Places); European Committee for Standardization: Brussels, Belgium, 2011. 